\title{
First Indication of Terrestrial Matter Effects on Solar Neutrino Oscillation
}

A. Renshaw ${ }^{7}$ K. Abe,${ }^{1,29}$ Y. Hayato,${ }^{1,29}$ K. Iyogi, ${ }^{1}$ J. Kameda, ${ }^{1,29}$ Y. Kishimoto, ${ }^{1,29}$ M. Miura,,${ }^{1,29}$ S. Moriyama, ${ }^{1,29}$ M. Nakahata, ${ }^{1,29}$ Y. Nakano, ${ }^{1}$ S. Nakayama, ${ }^{1,29}$ H. Sekiya, ${ }^{1,29}$ M. Shiozawa,,${ }^{1,29}$ Y. Suzuki, ${ }^{1,29}$ A. Takeda, ${ }^{1,29}$ Y. Takenaga ${ }^{1}$ T. Tomura, ${ }^{1,29}$ K. Ueno, ${ }^{1}$ T. Yokozawa, ${ }^{1}$ R. A. Wendell,,${ }^{1,29}$ T. Irvine, ${ }^{2}$ T. Kajita, ${ }^{2}, 29$ K. Kaneyuki, ${ }^{2,} 29$, K. P. Lee, ${ }^{2}$ Y. Nishimura,${ }^{2}$ K. Okumura, ${ }^{2,}{ }^{29}$ T. McLachlan, ${ }^{2}$ L. Labarga,${ }^{3}$ S. Berkman, ${ }^{4}$ H. A. Tanaka, ${ }^{4,31}$ S. Tobayama, ${ }^{4}$ E. Kearns,${ }^{5,29}$ J. L. Raaf, ${ }^{5}$ J. L. Stone,${ }^{5,29}$

L. R. Sulak,${ }^{5}$ M. Goldhabar,, , ${ }^{7}$ K. Bays,${ }^{7}$ G. Carminati, ${ }^{7}$ W. R. Kropp,${ }^{7}$ S. Mine, ${ }^{7}$ M. B. Smy,${ }^{7,29}$

H. W. Sobel,${ }^{7,29}$ K. S. Ganezer, ${ }^{8}$ J. Hill, ${ }^{8}$ W. E. Keig, ${ }^{8}$ N. Hong, ${ }^{9}$ J. Y. Kim,${ }^{9}$ I. T. Lim, ${ }^{9}$ T. Akiri, ${ }^{10}$ A. Himmel, ${ }^{10}$ K. Scholberg, ${ }^{10,29}$ C. W. Walter, ${ }^{10,29}$ T. Wongjirad,${ }^{10}$ T. Ishizuka, ${ }^{11}$ S. Tasaka, ${ }^{12}$ J. S. Jang, ${ }^{13}$ J. G. Learned, ${ }^{14}$ S. Matsuno, ${ }^{14}$ S. N. Smith, ${ }^{14}$ T. Hasegawa, ${ }^{15}$ T. Ishida,${ }^{15}$ T. Ishii, ${ }^{15}$ T. Kobayashi,,${ }^{15}$

T. Nakadaira, ${ }^{15}$ K. Nakamura, ${ }^{15,}{ }^{29}$ Y. Oyama, ${ }^{15}$ K. Sakashita, ${ }^{15}$ T. Sekiguchi,,${ }^{15}$ T. Tsukamoto, ${ }^{15}$ A. T. Suzuki, ${ }^{16}$ Y. Takeuchi, ${ }^{16}$ C. Bronner, ${ }^{17}$ S. Hirota,${ }^{17}$ K. Huang, ${ }^{17}$ K. Ieki,${ }^{17}$ M. Ikeda,${ }^{17}$ T. Kikawa, ${ }^{17}$ A. Minamino, ${ }^{17}$ T. Nakaya, ${ }^{17}{ }^{29}$ K. Suzuki, ${ }^{17}$ S. Takahashi,${ }^{17}$ Y. Fukuda, ${ }^{18}$ K. Choi, ${ }^{19}$ Y. Itow,${ }^{19}$ G. Mitsuka, ${ }^{19}$ P. Mijakowski,${ }^{35}$ J. Hignight ${ }^{20}$ J. Imber, ${ }^{20}$ C. K. Jung, ${ }^{20}$ C. Yanagisawa, ${ }^{20}$ H. Ishino, ${ }^{21}$ A. Kibayashi, ${ }^{21}$ Y. Koshio,${ }^{21}$

T. Mori, ${ }^{21}$ M. Sakuda,${ }^{21}$ T. Yano, ${ }^{21}$ Y. Kuno, ${ }^{22}$ R. Tacik, ${ }^{23,}{ }^{32}$ S. B. Kim,${ }^{24}$ H. Okazawa, ${ }^{25}$ Y. Choi, ${ }^{26}$ K. Nishijima, ${ }^{27}$ M. Koshiba, ${ }^{28}$ Y. Totsuka, ${ }^{28, *}$ M. Yokoyama, ${ }^{28,}{ }^{29}$ K. Martens,${ }^{29}$ Ll. Marti, ${ }^{29}$ M. R. Vagins, ${ }^{29,7}$ J. F. Martin, ${ }^{30}$ P. de Perio, ${ }^{30}$ A. Konaka, ${ }^{32}$ M. J. Wilking, ${ }^{32}$ S. Chen, ${ }^{33}$ Y. Zhang, ${ }^{33}$ and R. J. Wilkes ${ }^{34}$

(The Super-Kamiokande Collaboration)

\footnotetext{
${ }^{1}$ Kamioka Observatory, Institute for Cosmic Ray Research, University of Tokyo, Kamioka, Gifu 506-1205, Japan ${ }^{2}$ Research Center for Cosmic Neutrinos, Institute for Cosmic Ray Research, University of Tokyo, Kashiwa, Chiba 277-8582, Japan

${ }^{3}$ Department of Theoretical Physics, University Autonoma Madrid, 28049 Madrid, Spain

${ }^{4}$ Department of Physics and Astronomy, University of British Columbia, Vancouver, BC, V6T1Z4, Canada

${ }^{5}$ Department of Physics, Boston University, Boston, MA 02215, USA

${ }^{6}$ Physics Department, Brookhaven National Laboratory, Upton, NY 11973, USA

${ }^{7}$ Department of Physics and Astronomy, University of California, Irvine, Irvine, CA 92697-4575, USA

${ }^{8}$ Department of Physics, California State University, Dominguez Hills, Carson, CA 90747, USA

${ }^{9}$ Department of Physics, Chonnam National University, Kwangju 500-757, Korea

${ }^{10}$ Department of Physics, Duke University, Durham NC 27708, USA

${ }^{11}$ Junior College, Fukuoka Institute of Technology, Fukuoka, Fukuoka 811-0295, Japan

${ }^{12}$ Department of Physics, Gifu University, Gifu, Gifu 501-1193, Japan

${ }^{13}$ GIST College, Gwangju Institute of Science and Technology, Gwangju 500-712, Korea

${ }^{14}$ Department of Physics and Astronomy, University of Hawaii, Honolulu, HI 96822, USA

${ }^{15}$ High Energy Accelerator Research Organization (KEK), Tsukuba, Ibaraki 305-0801, Japan

${ }^{16}$ Department of Physics, Kobe University, Kobe, Hyogo 657-8501, Japan

${ }^{17}$ Department of Physics, Kyoto University, Kyoto, Kyoto 606-8502, Japan

${ }^{18}$ Department of Physics, Miyagi University of Education, Sendai, Miyagi 980-0845, Japan

${ }^{19}$ Solar Terrestrial Environment Laboratory, Nagoya University, Nagoya, Aichi 464-8602, Japan

${ }^{20}$ Department of Physics and Astronomy, State University of New York at Stony Brook, NY 11794-3800, USA

${ }^{21}$ Department of Physics, Okayama University, Okayama, Okayama 700-8530, Japan

${ }^{22}$ Department of Physics, Osaka University, Toyonaka, Osaka 560-0043, Japan

${ }^{23}$ Department of Physics, University of Regina, 3737 Wascana Parkway, Regina, SK, S4SOA2, Canada

${ }^{24}$ Department of Physics, Seoul National University, Seoul 151-742, Korea

${ }^{25}$ Department of Informatics in Social Welfare, Shizuoka University of Welfare, Yaizu, Shizuoka, 425-8611, Japan

${ }^{26}$ Department of Physics, Sungkyunkwan University, Suwon 440-746, Korea

${ }^{27}$ Department of Physics, Tokai University, Hiratsuka, Kanagawa 259-1292, Japan ${ }^{28}$ The University of Tokyo, Bunkyo, Tokyo 113-0033, Japan

${ }^{29}$ Kavli Institute for the Physics and Mathematics of the Universe (WPI), Todai

Institutes for Advanced Study, University of Tokyo, Kashiwa, Chiba 277-8582, Japan

${ }^{30}$ Department of Physics, University of Toronto, 60 St., Toronto, Ontario, M5S1A7, Canada

${ }^{31}$ Institute of Particle Physics, Canada, University of Toronto, 60 Saint George St., Toronta, ON, M5S1A7, Canada

${ }^{32}$ TRIUMF, 4004 Wesbrook Mall, Vancouver, BC, V6T2A3, Canada

${ }^{33}$ Department of Engineering Physics, Tsinghua University, Beijing, 100084, China

${ }^{34}$ Department of Physics, University of Washington, Seattle, WA 98195-1560, USA

${ }^{35}$ National Centre For Nuclear Research, 00-681 Warsaw, Poland
}

(Dated: August 27, 2018) 


\begin{abstract}
We report an indication that the elastic scattering rate of solar ${ }^{8} \mathrm{~B}$ neutrinos with electrons in the Super-Kamiokande detector is larger when the neutrinos pass through the Earth during nighttime. We determine the day/night asymmetry, defined as the difference of the average day rate and average night rate divided by the average of those two rates, to be $(-3.2 \pm 1.1$ (stat) \pm 0.5 (syst) $) \%$, which deviates from zero by $2.7 \mathrm{\sigma}$. Since the elastic scattering process is mostly sensitive to electronflavored solar neutrinos, a non-zero day/night asymmetry implies that the flavor oscillations of solar neutrinos are affected by the presence of matter within the neutrinos' flight path. SuperKamiokande's day/night asymmetry is consistent with neutrino oscillations for $4 \times 10^{-5} \mathrm{eV}^{2} \leq$ $\Delta m_{21}^{2} \leq 7 \times 10^{-5} \mathrm{eV}^{2}$ and large mixing values of $\theta_{12}$, at the $68 \%$ C.L.
\end{abstract}

Neutrino flavor oscillations occur when the phase difference of a superposition of massive neutrinos changes. Such phase changes occur while neutrinos are propagating in vacuum (vacuum oscillations). Wolfenstein [1] realized that the neutrino-electron elastic forward-scattering amplitude introduces additional phase shifts. As a consequence, neutrinos propagating in matter will oscillate differently than neutrinos propagating through vacuum. These matter effects are a fundamental prediction of the present theory of neutrino oscillations. In this letter, we report an indication of the existence of such matter effects.

Vacuum oscillations cannot easily explain a solar neutrino electron-flavor survival probability $P_{e e}$ which is measured to be significantly below one half [2 8$]$, in the energy region of $\sim 8$ to $18 \mathrm{MeV}$. Mikheyev and Smirnov [9] explained this experimental fact as the adiabatic transformation of the neutrinos through the varying solar density causing a resonant conversion to the second mass eigenstate inside the Sun (MSW resonance). Lower energy $(<2 \mathrm{MeV})$ solar neutrino data [10] are still described well as averaged vacuum oscillations. However, searches for the transition of $P_{e e}$ from the MSW resonance to vacuum oscillations (near $3 \mathrm{MeV}$ ) were so far unsuccessful [8, 11]. Moreover, these previous observations imply matter effects only indirectly, since there is no "control beam" of solar neutrinos that only propagates in vacuum. Atmospheric neutrino experiments can probe the existence of matter effects within the Earth in a similar fashion, and while there is currently no significant departure of present atmospheric data [12, 13] from the vacuum oscillation predictions, these effects will serve to determine the mass hierarchy and $\mathrm{CP}$ phase in future atmospheric and long baseline experiments.

The cleanest and most direct test of matter effects on neutrino oscillations is the comparison of the daytime and the nighttime solar neutrino interaction rates (solar day/night effect). In this comparison, the solar zenith angle controls the size and length of the terrestrial matter density through which the neutrinos pass, and thereby the oscillation probability and the observed interaction rate. An increase in the nighttime interaction rates implies a regeneration of electron-flavor neutrinos. Other solar neutrino measurements [3, 8, 14] have found no significant day/night differences. Here, we report a $2.7 \sigma$ indication of a non-zero solar neutrino day/night effect 1

Super-Kamiokande (SK) is a 50,000 metric ton cylindrical water Cherenkov detector. The optically separated 32,000 ton inner detector (ID), viewed by $\sim 11,10050 \mathrm{~cm}$ diameter photomultiplier tubes (PMTs), is surrounded by an 18,000 ton active veto shield, viewed by $\sim 1,900$ $20 \mathrm{~cm}$ diameter PMTs. The detector is described in detail in [15]. SK detects recoil electrons coming from the elastic scattering of solar neutrinos with electrons. Only ${ }^{8} \mathrm{~B}$ and hep solar neutrinos produce recoil electrons of sufficiently high energy to be detected in SK. Neutrino-electron elastic scattering is mostly sensitive to electron-flavored neutrinos, because the cross section for $\nu_{\mu, \tau}$ scattering is six times smaller, since only the electroweak neutral-current interaction channel contributes. The scattering vertex is reconstructed using the timing of the Cherenkov light, while the direction and energy of the recoil electrons are determined from the light pattern and intensity. For $10 \mathrm{MeV}$ electrons in SK-IV, the vertex resolution is $52 \mathrm{~cm}$, the directional resolution is $25^{\circ}$ (limited by multiple Coulomb scattering), and the energy resolution is $14.0 \%$ (dominated by Poisson fluctuations of the number of photons detected with $\sim 6$ photo-electrons per $\mathrm{MeV})$. More details are given in [4 6 ,, 11$]$.

There are four distinct phases of SK. Initially, SK-I had 11,146 ID PMTs and used 1,496 live days between 1996 and 2001 for low-energy analysis [4]. In 2001, an accident during maintenance destroyed $\sim 7,000$ ID PMTs. 5,182 surviving and spare ID PMTs were then deployed for SK-II [5], running between 2002-2005, with a total low-energy analysis livetime of 791 days. After full reconstruction of the detector, SK-III [] $]$ took data between 2005 and 2008, acquiring 548 live days for the low-energy analysis, with 11,129 ID PMTs. Since 2008, SK-IV has been running with upgraded electronics and data acquisition system (DAQ). In this paper, data from SK-I, SK-II, SK-III and 1,306 live days of SK-IV are used [11]. The day/night analysis uses recoil electrons above $4.5 \mathrm{MeV}$ in SK-I, III, and IV and $7 \mathrm{MeV}$ in SK-II. The energy range of the SK-I-III day/night analysis is identical as in [4-6]; however, here it is quoted as kinetic energy (we simply

\footnotetext{
1 This is consistent with the strong limits of [14] using $861 \mathrm{keV}$ mono-energetic neutrinos, an energy where presently-preferred neutrino oscillation parameters predict no day/night effect.
} 
subtracted $0.5 \mathrm{MeV}$ ) while [4 $[\underline{6}]$ use total energy. In [11], the observed solar neutrino signal at lower recoil electron energies is used for the flux and spectrum analysis.

At the time of each event, the solar zenith angle $\theta_{z}$ is determined. This is the angle between the vector from the solar position to the event vertex and the vertical detector $(z)$ axis. The precision of the cosine of this angle is much better than $10^{-3}$, the bin width used in the following analysis. The accuracy of this angle is limited only by SK's absolute time precision (a few $100 \mathrm{~ns}$ ) and basic astronomy. The SK elastic scattering rate as a function of the solar zenith angle $r\left(\cos \theta_{z}\right)$ is used to search for a day/night difference in the interaction rate. The expected change in the interaction rate due to the varying Sun-Earth distance (induced by the eccentricity of Earth's orbit) is taken into account throughout this paper. The most straight-forward method to look for a day/night effect is to define separate day $\left(\cos \theta_{z} \leq 0\right)$ and night $\left(\cos \theta_{z}>0\right)$ samples. Based on $r_{D}\left(r_{N}\right)$, the average scattering rate of the day (night) sample, we define the SK day/night asymmetry as $A_{\mathrm{DN}}=\left(r_{D}-r_{N}\right) / \frac{1}{2}\left(r_{D}+r_{N}\right)$. Therefore, $A_{\mathrm{DN}}=0$ implies no terrestrial matter effect on solar neutrino oscillations.

To increase sensitivity, 16] first introduced an unbinned maximum likelihood fit of the solar zenith angle distribution of the rate $r\left(\cos \theta_{z}\right)$ to the day/night variation amplitude $\alpha$. This was done using "shapes" of such variations expected from neutrino oscillation calculations. By construction, $\alpha$ scales the calculated day/night asymmetry $A_{\mathrm{DN}}^{\text {calc }}$ while leaving the average rate unchanged, giving the measured day/night asymmetry $A_{\mathrm{DN}}^{\text {fit }}=A_{\mathrm{DN}}^{\text {calc }} \times \alpha$. This more sophisticated method, referred to as the "amplitude fit", was also used in [4]. The calculated oscillation shapes ignore the SK daytime overburden, which can be up to a few kilometers depending on the solar zenith angle.

We refer to the angle between the solar and reconstructed recoil electron candidate directions as $\theta_{\text {sun }}$. The solar neutrino interaction rate is extracted by an extended maximum likelihood fit [4] to the $\cos \theta_{\text {sun }}$ distribution. [16] expands the signal likelihood to allow for a time-dependent solar neutrino-electron elastic scattering rate, parameterized by the amplitude scaling variable $\alpha$. The best-fit $\alpha$, multiplied with $A_{\mathrm{DN}}^{\text {calc }}$, defines a best-fit $A_{\mathrm{DN}}^{\mathrm{fit}}$. In this manner the day/night asymmetry is measured more precisely statistically. It is also less vulnerable to some key systematic effects, such as directional variation of the energy scale (the frequency of which is limited by SK's angular resolution, $\sim 25^{\circ}$ ).

Because the amplitude fit depends on the shape of the day/night variation, given for each energy bin in 16] (and also in [4]), it necessarily depends on the assumed oscillation parameters. Vacuum oscillations depend on the neutrino energy, the length of the flight path $L$ and the oscillation parameters; the difference of the squared

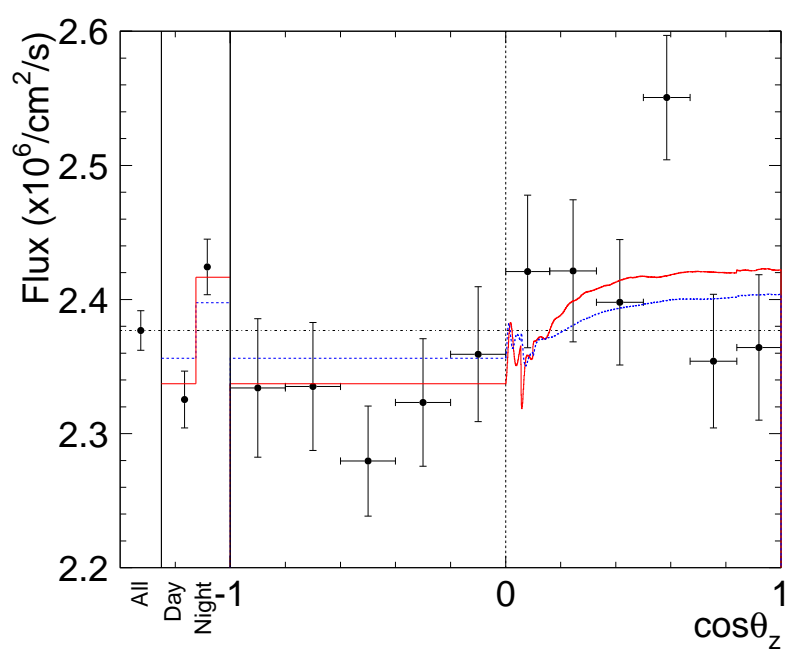

FIG. 1. SK combined solar zenith angle dependence of the ${ }^{8} \mathrm{~B}$ solar neutrino flux. Solid red (dashed blue) gives the prediction based on oscillation parameters from a fit to SK data while constraining the flux (solar+KamLAND fit) and the dashed-dotted line gives the total average flux.

masses of the mass eigenstates $\Delta m_{i j}^{2}(i=1,2,3, \ldots)$ and the mixing of the mass eigenstates with the flavor eigenstates (mixing angles $\theta_{i j}$ ). If the neutrinos propagate through matter, then the density of the matter will effectively change the oscillation parameters. It was shown analytically by 17 19] that $r\left(\cos \theta_{z}\right)-r_{D}$ oscillates with the vacuum frequency $\frac{\Delta m_{21}^{2}}{4 E} L$, if $L \approx 2 R \cos \theta_{z}$ denotes now the path length of the neutrino inside the Earth $(R$ being the Earth radius). Although the dependence of matter effects on the mixing angles (in or near the large mixing angle solutions and for $\theta_{13}$ values consistent with reactor neutrino measurements [20]) is quite small, the dependence on $\Delta m_{21}^{2}$ is more noticeable. The fit is run for solar oscillation parameter sets which predict various matter effects $\left(10^{-9} \mathrm{eV}^{2} \leq \Delta m_{21}^{2} \leq 10^{-3} \mathrm{eV}^{2}\right.$ and $10^{-4} \leq \sin ^{2} \theta_{12} \leq 1$ ), for values of $\sin ^{2} \theta_{13}$ between 0.015 and 0.035 .

Fig. 1 combines the data from all four SK phases to show the measured zenith angle distribution of the flux assuming no oscillations. The expected zenith variation assuming best-fit oscillation parameters 11 from a global fit based on solar neutrino data 2, 4, 6, 8, 10, 11] is overlaid in solid red. The dashed blue line also includes reactor anti-neutrino data [22]. The day and night flux values given in the left portion of Fig. 1 imply a day/night asymmetry of $A_{\mathrm{DN}}=(-4.2 \pm 1.2$ (stat) $) \%$. The ninth data point in the right panel of Fig. 1 shows an $\sim 2$ sigma deviation, enhancing the resulting $A_{D N}$. We believe this is a statistical flucuation, accounted for by the quoted statistical uncertainties. The result $A_{D N}^{\mathrm{fit}}$ coming from the unbinned maximum likelihood fit is less prone to these types of flucuations. To calculate the total systematic uncertainty, the individual systematic uncertainties 
TABLE I. Day/night asymmetry for each SK phase, coming from separate day and night rate measurements (middle column) and the amplitude fit (right column). The uncertainties shown are statistical and systematic. The entire right column assumes the SK best-fit point of oscillation parameters.

\begin{tabular}{lcc}
\hline \hline & $A_{\mathrm{DN}} \pm($ stat $) \pm($ syst $)$ & $A_{\mathrm{DN}}^{\mathrm{fit}} \pm$ (stat) \pm (syst) \\
\hline SK-I & $(-2.1 \pm 2.0 \pm 1.3) \%$ & $(-2.0 \pm 1.7 \pm 1.0) \%$ \\
SK-II & $(-5.5 \pm 4.2 \pm 3.7) \%$ & $(-4.3 \pm 3.8 \pm 1.0) \%$ \\
SK-III & $(-5.9 \pm 3.2 \pm 1.3) \%$ & $(-4.3 \pm 2.7 \pm 0.7) \%$ \\
SK-IV & $(-5.3 \pm 2.0 \pm 1.4) \%$ & $(-3.4 \pm 1.8 \pm 0.6) \%$ \\
\hline Combined & $(-4.2 \pm 1.2 \pm 0.8) \%$ & $(-3.2 \pm 1.1 \pm 0.5) \%$ \\
\hline \hline
\end{tabular}

of the four phases (for values see [4-6, 11]) are assumed to be uncorrelated, since the dominant contributions come from the energy-scale uncertainty (tuned independently for each phase) and the background directional distribution shape uncertainty (evaluated from detector zenith angle data distributions and limited by statistical fluctuations). The measured day/night asymmetry when using this simple method is shown in the middle column of Table I along with the statistical and systematic uncertainties. SK measures the day/night asymmetry in this simple way as $A_{\mathrm{DN}}=(-4.2 \pm 1.2$ (stat) \pm 0.8 (syst) $) \%$, which deviates from zero by $2.8 \sigma$.

Fig. 2 shows the combined SK-I/II/III/IV day/night amplitude fit as a function of recoil electron energy. In each recoil electron energy bin $e$, the day/night variation is fit to an amplitude $\alpha_{e}$. The displayed day/night asymmetry values are the product of the fit amplitude $\alpha_{e}$ with the expected day/night asymmetry $A_{\mathrm{DN}, \text { calc }}^{e}$ (red), when using the SK best-fit point of oscillation parameters $\left(\Delta m_{21}^{2}=4.8_{-0.9}^{+1.8} \times 10^{-5} \mathrm{eV}^{2}, \sin ^{2} \theta_{12}=0.342_{-0.025}^{+0.029}\right.$ [11] and $\sin ^{2} \theta_{13}=0.025 \pm 0.003$ [20]). These parameters are chosen when using SK's spectral and time variation data along with constraints on the ${ }^{8} \mathrm{~B}$ solar neutrino flux and $\theta_{13}$. When all energy bins are fit together and the same oscillation parameters assumed, the resulting SKmeasured day/night asymmetry coming from the amplitude fit is $A_{\mathrm{DN}}^{\text {fit }}=(-3.2 \pm 1.1$ (stat) $) \%$, with an asymmetry of $-3.3 \%$ expected by numerical calculations (see [16] for details).

Originally the systematic uncertainties on the SK-I and II day/night amplitude measurements (see [16]) were con-

TABLE II. Day/night amplitude fit systematic uncertainties by SK phase. The total is found by adding the contributions for each phase in quadrature.

\begin{tabular}{lcccc}
\hline \hline & SK-I & SK-II & SK-III & SK-IV \\
\hline Energy Scale & $0.8 \%$ & $0.8 \%$ & $0.2 \%$ & $0.05 \%$ \\
Energy Resolution & $0.05 \%$ & $0.05 \%$ & $0.05 \%$ & $0.05 \%$ \\
Background Shape & $0.6 \%$ & $0.6 \%$ & $0.6 \%$ & $0.6 \%$ \\
Event Selection & - & - & $0.2 \%$ & $0.1 \%$ \\
Earth Model [21] & $0.01 \%$ & $0.01 \%$ & $0.01 \%$ & $0.01 \%$ \\
\hline Total & $1.0 \%$ & $1.0 \%$ & $0.7 \%$ & $0.6 \%$ \\
\hline \hline
\end{tabular}

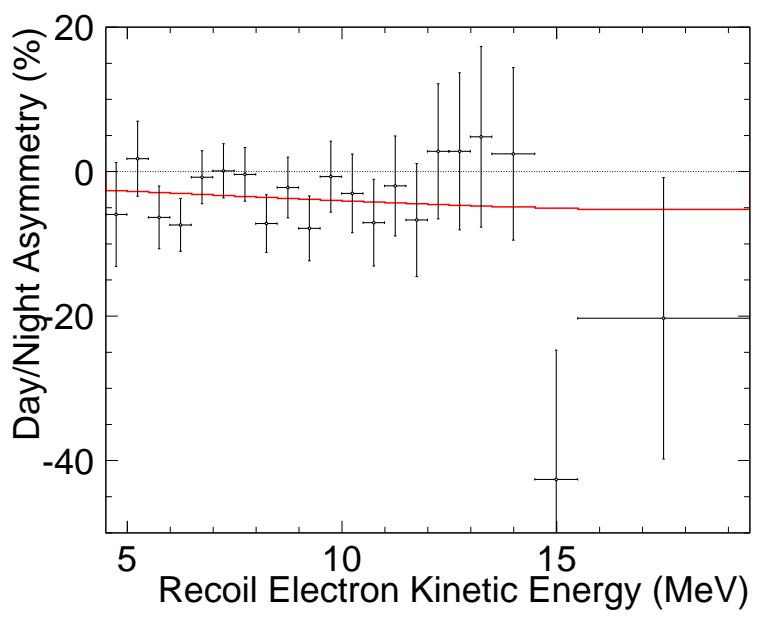

FIG. 2. SK day/night amplitude fit as a function of recoil electron kinetic energy (unlike [16] which uses total energy), shown as the measured amplitude times the expected day/night asymmtery, for oscillation parameters chosen by the SK best-fit. The error bars shown are statistical uncertainties only and the expected dependence is shown in red.

servatively assigned to be the same as that of the simple day/night asymmetry measurement (see [4, 5]). Because [4, 5] only give total systematic uncertainties and not those for each of the components, we have now reestimated the systematic uncetainties of the day/night amplitude fit of the first two SK phases, using similar methods as for SK-III and IV. The methods for estimating the systematic uncertainties of the amplitude fit in SK-III and IV are detailed in [11] (see Section 9.3). A summary of the various components of the systematic uncertainty on the day/night amplitude fit, as well as the total, is given in Table II for each SK phase.

During the SK-I and II phases, the largest contribution to the systematic uncertainty came from the directional dependence of the energy scale. From the beginning of the SK-III phase, a depth-dependent water transparency parameter was introduced into the MC simulation program. This corrects for the depth-dependence of the water absorption coefficient and greatly reduces the directional dependence of the energy scale. The further reduction seen from SK-III to SK-IV comes from an improvement in the comparison between data and MC timing, the result of the electronics upgrade prior to SK-IV. The largest contribution to the systematic uncertainty now comes from the expected background shapes, which are derived from fits to the detector's zenith and azimuthal angle distributions after statistical subtraction of the solar neutrinos. The accuracy of these shapes are limited by statistics.

The additional contribution to the systematic uncertainty during SK-III and IV, coming from the event selection, is the result of the combination of the external 
event and tight fiducial volume cuts (see [6, 11]). The tight fiducial volume cut introduced at the start of SKIII is asymmetric in the $z$ direction, causing the external event cut to have different selection efficiencies during the day and night times. As for the case of the simple day/night asymmetry measurement, the total systematic uncertainty of each SK phase is assumed to be uncorrelated, and is added in quadrature to the statistical uncertainty of the corresponding phase before combining the results of each phase together.

The right column of Table [I lists the measured day/night asymmetry coming from the amplitude fit to each phase, as well as the combined fit, for oscillations parameters at the SK best-fit point. The combined fit takes into account energy threshold and resolution. The equivalent SK day/night asymmetry coming from the amplitude fit is

$$
A_{\mathrm{DN}}^{\mathrm{fit}}=(-3.2 \pm 1.1(\text { stat }) \pm 0.5(\text { syst })) \%,
$$

which differs from zero by $2.7 \sigma$. The measured value of the day/night asymmetry agrees with $-3.2 \%$, within $\pm 0.2 \%$, for all sets of oscillation parameters contained in the large mixing angle region. The SNO experiment also performed a search for the day/night variation of the charged-current interaction rate on deuterium [8]. From their result, we predict the SK day/night asymmetry to be $A_{\mathrm{DN}}^{\text {pred }}=(-2.0 \pm 1.8) \%$ 2 . Combining SK and SNO data yields $A_{\mathrm{DN}}^{\mathrm{fit}}=(-2.9 \pm 1.0($ stat+syst $)) \%$, which differs from zero by $2.9 \sigma$. The expected SK day/night asymmetry for these oscillation parameters is $-3.3 \%$. Changing $\Delta m_{21}^{2}$ to $7.41 \times 10^{-5} \mathrm{eV}^{2}$ and $\sin ^{2} \theta_{12}$ to 0.31 (motivated by KamLAND data [22]) changes the SK-measured day/night asymmetry to $(-3.0 \pm 1.0$ (stat) \pm 0.5 (syst) $) \%$, slightly reducing the significance for a non-zero day/night asymmetry from 2.7 to $2.6 \sigma$.

Fig. 3 shows the $\Delta m_{21}^{2}$ dependence of the equivalent day/night asymmetry of the SK combined amplitude fit for $\sin ^{2} \theta_{12}=0.314$ and $\sin ^{2} \theta_{13}=0.025$. The expected day/night asymmetry is indicated by the red curve. The point where the best-fit crosses the expected curve represents the value of $\Delta m_{21}^{2}$ where the measured day/night amplitude is $\alpha=1$. Superimposed are the $1 \sigma$ allowed ranges in $\Delta m_{21}^{2}$ from the solar global fit [11] (green) and from the KamLAND experiment 22] (blue). The amplitude fit has negligible dependence on the values of $\theta_{12}$ (within the large mixing angle region of oscillation parameters) and $\theta_{13}\left(0.015 \leq \sin ^{2} \theta_{13} \leq 0.035\right)$, leading to a $68 \%$ C.L. allowed range of $4 \times 10^{-5} \mathrm{eV}^{2} \leq \Delta m_{21}^{2} \leq$

\footnotetext{
${ }^{2}$ SNO actually models the night/day asymmetry of the survival probability as $a_{0}+a_{1}\left(E_{\nu}-10 \mathrm{MeV}\right)$ and fits the coefficients $a_{i}$ [8]. We scale the expected coefficients (based on $\Delta m_{21}^{2}=$ $4.84 \times 10^{-5} \mathrm{eV}^{2}$ ) by an amplitude $\alpha_{\mathrm{SNO}}$ and minimize the SNO $\chi^{2}$ with respect to it. SNO data then implies $a_{0}=(3.4 \pm 2.9) \%$, while SK and SNO combined is equivalent to $a_{0}=(4.8 \pm 1.6) \%$
}

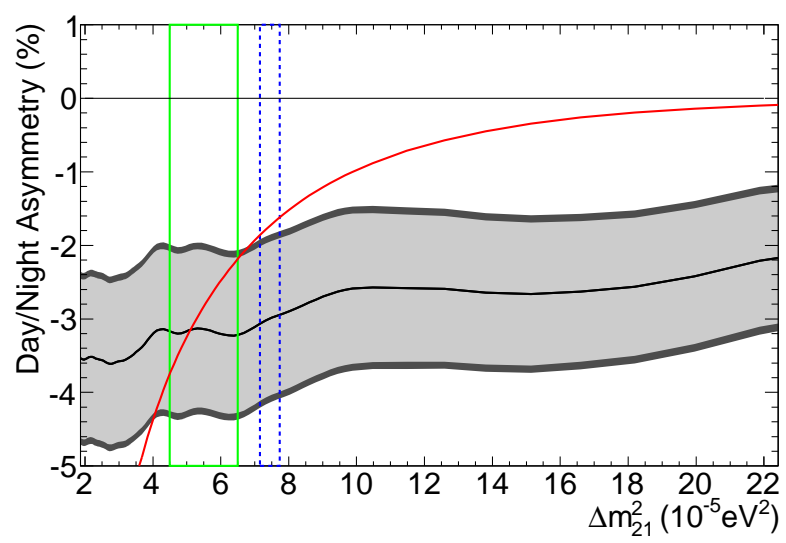

FIG. 3. Dependence of the measured day/night asymmetry (fitted day/night amplitude times the expected day/night asymmetry (red)) on $\Delta m_{21}^{2}$, for $\sin ^{2} \theta_{12}=0.314$ and $\sin ^{2} \theta_{13}=0.025$. The $1 \sigma$ statistical uncertainties are given by the light gray band. The additional dark gray width to the band shows the inclusion of the systematic uncertainties. Overlaid are the $1 \sigma$ allowed ranges from the solar global fit (solid green) and the KamLAND experiment (dashed blue).

$7 \times 10^{-5} \mathrm{eV}^{2}$ (as shown in Fig. 3). Aside from the amplitude of the day/night asymmetry, another handle to $\Delta m_{21}^{2}$ is the day/night variation frequency. Although the amplitude of the day/night variation is too small (compared to present uncertainties) to measure the frequency, some frequencies are favored by about $2 \sigma$ over others.

Even so, the neutrino flux-independent solar neutrino oscillation analysis of [16] uses frequency and amplitude of the day/night variation as well as spectral information. We calculate the $\log$ likelihood ratio between $\alpha=1$ and $\alpha=0$, multiply by -2 , and then add it to the $\chi^{2}$ values of the fit to the recoil electron spectrum (see [11]). Fig. [4 shows the flux-independent SK-I/II/III/IV contours of $68 \%$ (solid thin line), $95 \%$ (solid thick line), three sigma (dashed-dotted line), and five sigma (dashed gray line) significance. For the $95 \%$ C.L., regions preferred by the day/night variation data are highlighted in gray. In the case of $68 \%$ C.L., those regions closely match the two lower $\Delta m_{21}^{2} 68 \%$ contours shown in the figure. The black asterisk marks the parameters selected by all solar neutrino [8, 10, 11, 23] and KamLAND data [22], including this work. The SK flux-independent contours agree with those parameters within two sigma. The previously suggested low (small mixing angle) solution of neutrino oscillation parameters is excluded at more than four (five) sigma 3 .

In conclusion, we find an indication of electron-flavor regeneration in solar neutrino oscillations due to the pres-

\footnotetext{
3 The Low and small mixing angle solution parameters can be seen in [8] and [24], respectively.
} 


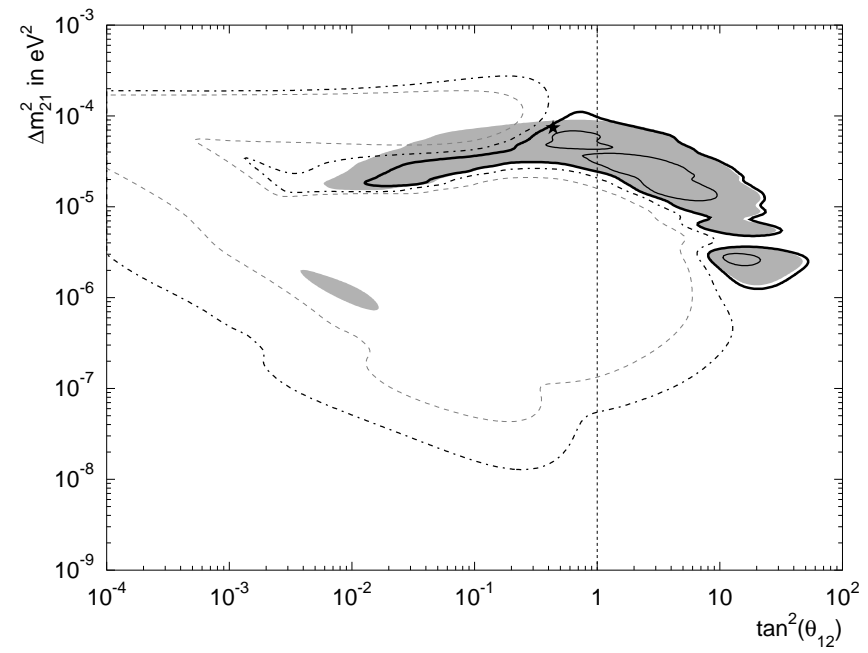

FIG. 4. Contours at $68 \%$ (solid thin line), 95\% (solid thick line), three sigma (dashed-dotted line), and five sigma (dashed gray line) of the flux-independent SK solar neutrino oscillation analysis. The shaded gray area indicates regions preferred by the day/night variation data for the $95 \%$ case. The best-fit parameters resulting from a fit to all solar neutrino 8, 10, 11, 23] and KamLAND 22] data is shown by the black asterisk.

ence of terrestrial matter effects. The fit amplitude of the solar zenith angle variation of the SK solar neutrino interaction rate corresponds to a day/night asymmetry of $(-3.2 \pm 1.1$ (stat) \pm 0.5 (syst) $) \%$, which deviates from zero by $2.7 \sigma$. This analysis probes matter effects directly, since it compares the flavor content of the solar neutrino beam with Earth matter to that without. Therefore, this is the first direct indication that neutrino oscillation probabilities are modified by the presence of matter.

The authors gratefully acknowledge the cooperation of the Kamioka Mining and Smelting Company. Super-K has been built and operated from funds provided by the Japanese Ministry of Education, Culture, Sports, Science and Technology, the U.S. Department of Energy, and the U.S. National Science Foundation. This work was partially supported by the Research Foundation of Korea (BK21 and KNRC), the Korean Ministry of Science and Technology, the National Science Foundation of China (Grant NO. 11235006), the European Union FP7 ITN INVISIBLES (Marie Curie Actions, PITN-GA2011-289442) and the State Committee for Scientific Research in Poland.
* Deceased.

[1] L. Wolfenstein, Phys. Rev. D 17, 2369 (1978).

[2] R. Davis, Jr. et al., Phys. Rev. Lett. 20, 1205 (1968).

[3] Y. Fukuda et al., Phys. Rev. Lett. 77, 1683 (1996).

[4] J. Hosaka et al., Phys. Rev. D 73, 112001 (2006).

[5] J. P. Cravens et al., Phys. Rev. D 78, 032002 (2008).

[6] K. Abe et al., Phys. Rev. D 83, 052010 (2011).

[7] Q. R. Ahmad et al., Phys. Rev. Lett. 87, 071301 (2001).

[8] B. Aharmin et al., Phys. Rev. C 88, 025501 (2013).

[9] S. P. Mikheyev and A. Yu. Smirnov, Sov. Jour. Nucl. Phys. 42, 913 (1985).

[10] J. N. Abdurashitov et al. (SAGE collaboration), Phys. Rev. C 80, 015807 (2009); M. Altmann et al. (GALLEX Collaboration), Phys. Lett. B 616, 174 (2005); G. Bellini et al. (Borexino Collaboration), Phys. Rev. Lett. 107, 141302 (2011).

[11] A. Renshaw, "First Direct Evidence for Matter Enhanced Neutrino Oscillation, Using SuperKamiokande Solar Neutrino Data", Ph.D. Thesis, http://www-sk.icrr.u-tokyo.ac.jp/sk/pub/Renshaw_Doctoral_Th K. Abe et al., "Solar Neutrino Measurements in SK-IV", to be submitted to Phys. Rev. D (2014).

[12] J. Hosaka et al., Rhys. Rev. D 74, 032002 (2006).

[13] R. Wendell et al., Phys. Rev. D 81, 092004 (2010).

[14] G. Bellini et al., Phys. Lett. B 707 (2012).

[15] The SK Collaboration, Nucl. Insturm. Meth. A 501 (2003).

[16] M. B. Smy et al., Phys. Rev. D 69, 011104(R) (2004).

[17] A. N. Ioannisian and A. Y. Smirnov, Phys. Rev. Lett. 93, 241801 (2004).

[18] M. Blennow, T. Ohlsson and H. Snellman, Phys. Rev. D 69, 073006 (2004).

[19] E. K. Akhmedov, M. A. Tortola and J. W. F. Valle, JHEP 0405, 057 (2004).

[20] F. P. An et al. (Daya Bay Collaboration), Chin. Phys. C 37, 011001 (2013); J. K. Ahn et al. (RENO Collaboration), Phys. Rev. Lett. 108191802 (2012); Y. Abe et al. (Double Chooz Collaboration), Phys. Rev. D 86 052008 (2012); J. Beringer et al. (Paticle Data Group), Phys. Rev. D 86, 010001 (2012).

[21] A. M. Dziewonski and D. L. Anderson, Phys. Earth Planet. Inter. 25, 297 (1981); J. J. Durek and G. Ekstrom, Bull. Seism. Soc. Am. 86, 144-158 (1996).

[22] S. Abe et al., Phys. Rev. Lett. 100, 221803 (2008); The KamLAND Collaboration, arXiv:1303.4667v2 (2013).

[23] R. Davis et al. (Homestake Experiment), Phys. Rev. Lett. 20, 1205 (1968);

[24] M. C. Gonzalez-Garcia, Phys. Particles and Nucl. 42, 577 (2011). 